\title{
COMPLETELY CONTINUOUS COMPOSITION OPERATORS
}

\author{
JOSEPH A. CIMA AND ALEC MATHESON
}

\begin{abstract}
A composition operator $T_{b} f=f \circ b$ is completely continuous on $H^{1}$ if and only if $|b|<1$ a.e. If the adjoint operator $T_{b}^{*}$ is completely continuous on $V M O A$, then $T_{b}$ is completely continuous on $H^{1}$. Examples are given to show that the converse fails in general. Two results are given concerning the relationship between the complete continuity of an operator and of its adjoint in the presence of certain separability conditions on the underlying Banach space.
\end{abstract}

\section{INTRODUCTION}

There are sharp function theoretic criteria for determining when a holomorphic composition operator $T_{b}$ acting on the Hardy space $H^{p}$ is compact [Sh1], [Sh2]. It is also known that if such an operator is compact on one such space then it is compact on all the spaces [ST]. Recently J. H. Shapiro and C. Sundberg [SS] proved that if such a composition operator $T_{b}$ is compact on the Hardy space $H^{1}$ of the unit disk $\mathbb{D}$ then the operator $T_{b}$ is compact when considered as an operator on $L^{1}$ of the unit circle $\partial \mathbb{D}$. Using this fact, Sarason in [Sa2] proved that if a composition operator $T_{b}$ is weakly compact on $H^{1}$ then it is in fact a compact operator on this space. An operator $T$ on a Banach space $X$ is called completely continuous if it maps weakly convergent sequences to strongly convergent sequences. It is known that every compact operator is completely continuous [B, p. 143]. In some Banach spaces, including the reflexive spaces, every completely continuous operator is compact. A Banach space $X$ is said to have the Dunford-Pettis property if every weakly compact operator on $X$ is completely continuous. Because of this completely continuous operators are sometimes called Dunford-Pettis operators [Ro2], [B1], [B2]. In this paper we show that although the classes of completely continuous and compact composition operators agree on $H^{p}$ for $p>1$ they are not the same on $H^{1}$. It follows from Sarason's result that every weakly compact composition operator on $H^{1}$ is completely continuous, although it is known that $H^{1}$ does not have the Dunford-Pettis property [Di, p. 45].

It will be useful for us to consider the adjoint of $T_{b}$ and this leads us to a

Received by the editors October 5, 1993.

1991 Mathematics Subject Classification. Primary 47B38, 47B07; Secondary 30D55.

Key words and phrases. Composition operator, completely continuous operator.

Part of this work was done while the authors were visiting Montana State University and the authors thank them for their hospitality during this period. The second author would also like to thank Dale Alspach for several helpful discussions. 
characterization of the weakly convergent sequences in $V M O A$. The importance of the role of the adjoint is made clear by the following two results of Sarason [Sa1]. He proved that such a $T_{b}$ is compact on $L^{1}$ of the circle if and only if the adjoint $T_{b}^{*}$ maps $L^{\infty}$ into the continuous functions on the circle and that if the adjoint maps $L^{\infty}$ into $Q C$ (the bounded $V M O$ functions) then it maps $L^{\infty}$ into the continuous functions. One of the tools used in studying these problems is the close connection between compactness of $T$ and compactness of the adjoint (or similar properties relating the weak compactness of $T$ to certain range restrictions of $T^{* *}$ acting on the second adjoint). There is a paucity of such results for completely continuous operators. We prove one useful result connecting the behavior of completely continuous operators and their adjoints for separable Banach spaces.

We recommend the books of Conway [Con] and Diestel [Di] for good references to this material.

\section{Definitions}

Let $b$ be a holomorphic function mapping the unit disk into itself. Unless otherwise noted we will assume without loss of generality that $b(0)=0$. The holomorphic composition operator $T_{b}(f)(z)=f(b(z))$ will be denoted by $T$. It is a well-known consequence of Littlewood's subordination principle that $T$ is a bounded linear operator of norm one on the Hardy space $H^{1}$. The space $H^{1}$ is the dual of the space of holomorphic functions with vanishing mean oscillation written as $V M O A$ and the dual of $H^{1}$ is the space $B M O A$ of holomorphic functions with bounded mean oscillation. We note that $H^{1}$ is separable (and that $B M O A$ is not). We will denote the dual pairing between $f \in H^{1}$ and $\psi \in B M O A$ by $\langle f, \psi\rangle$ and note only that if $\psi$ is bounded, then this can be computed as a boundary integral

$$
\langle f, \psi\rangle=\frac{1}{2 \pi} \int_{-\pi}^{\pi} f\left(e^{i \theta}\right) \overline{\psi\left(e^{i \theta}\right)} d \theta .
$$

We will denote by $\|\phi\|_{*}$ the norm on $B M O A$ determined by its role as the dual space of $H^{1}$. We note that this is not the usual norm on $B M O A$, and, in particular, if $\phi \in B M O A \cap L^{\infty}$, then $\|\phi\|_{*} \leq\|\phi\|_{\infty}$, because $H^{1}$ is isometric to a subspace of $L^{1}$. Even though the adjoint $T^{*}$ acts on $B M O A$ it can also be considered as an operator on $V M O A$ and it is shown in [Sal] that $T^{*}$ restricted to $V M O A$ has range in $V M O A$, and hence $T$ on $H^{1}$ is the adjoint of this restriction. Finally, we will denote by $C$ the space of functions continuous on the unit circle $\partial \mathbb{D}$.

We recall the following facts concerning general Banach spaces. If $X$ is a Banach space and its topological dual $X^{*}$ is separable then the weak ${ }^{*}$ topology on the second dual $X^{* *}$ restricted to any closed ball is metric and hence one can deal with sequences when discussing convergence in this topology. Also if $X^{\prime}$ is the canonical injection of $X$ into $X^{* *}$ then $X^{\prime}$ with the weak ${ }^{*}$ topology agrees with $X$ with its weak topology. This implies (for $X^{*}$ separable) that every bounded sequence in $X$ has a weak Cauchy subsequence.

\section{COMPlete CONTINUity ON $H^{1}$}

We begin with a characterization of complete continuity for holomorphic composition operators on $H^{1}$. 
Proposition 1. The composition operator $T$ is completely continuous on $H^{1}$ if and only if $\left|b\left(e^{i t}\right)\right|<1$ a.e. on the unit circle.

Proof. Assume first that $T$ is completely continuous on $H^{1}$. It is a consequence of the Riemann-Lebesgue Lemma that the sequence $\left(z^{n}\right)$ is weakly null in $H^{1}$. Hence $\left\|b^{n}\right\|_{1}$ tends to 0 , which is impossible if $b$ has modulus one on a set of positive measure.

Conversely, suppose that $|b|<1$ a.e. Let $\left(f_{n}\right)$ be a weakly null sequence in $H^{1}$. Then $\left(f_{n}\right)$ tends to zero pointwise in the unit disk and so $\left(f_{n} \circ b\right)$ tends to zero pointwise a.e. on the circle. Since $\left(f_{n} \circ b\right)$ is also weakly null, it follows from a theorem of Dunford and Pettis [DP, p. 295] that $\left\|f_{n} \circ b\right\|_{1}$ tends to zero. Hence $T$ is completely continuous.

Remark 1 . Note that $b(z)=z\left(\frac{z+1}{2}\right)$ induces a completely continuous operator on $H^{1}$ but the operator is not compact.

It is easy to see that if $T$ is completely continuous on $H^{1}$ then the adjoint $T^{*}$ considered as a map on $V M O A$ maps $V M O A$ into itself and we are interested in whether or not the complete continuity of $T$ implies that of $T^{*}$ on $V M O A$. It is important to understand the weakly convergent sequences in $V M O A$ so we first characterize the weakly convergent sequences in $V M O A$. We need a simple consequence of the normality of the unit ball in $B M O A$.

Lemma 1. Let $\left(\psi_{n}\right)$ be a bounded sequence in BMOA which converges weak* to $\psi$. Then $\left(\psi_{n}\right)$ converges to $\psi$ uniformly on compact subsets of the unit disk. Proof. It suffices to assume that $\psi=0$ and that $\left\|\psi_{n}\right\|_{*} \leq 1$ for all $n$. Let $g_{z}(\zeta)=\frac{1}{1-\zeta \bar{z}}$, so that $\psi(z)=\left\langle\psi, g_{z}\right\rangle$. Clearly $\left\|g_{z}\right\|_{1} \leq \frac{1}{1-|z|}$, so that for each compact $K \subset \mathbb{D}$ there is a constant $C_{K}$ such that $|\psi(z)| \leq C_{K}\|\psi\|_{*}$ for all $\psi \in B M O A$. Now for compact $K \subset \mathbb{D}$, let $\delta=\operatorname{dist}(K, \partial \mathbb{D})$. Then the sequence $\left(\psi_{n}\right)$ is uniformly bounded for $|z| \leq 1-\frac{1}{2 \delta}$ and converges pointwise to 0 on $K$. Hence $\left(\psi_{n}\right)$ converges uniformly to 0 on $K$ by Montel's Theorem.

Theorem 1. The sequence $\left(\psi_{n}\right)$ in VMOA is weakly null if and only if it is bounded and converges to zero uniformly on compact subsets of the unit disk.

Proof. It is clear that $\left(\psi_{n}\right)$ is weakly null if and only if it is bounded and converges weak ${ }^{*}$ to zero as a sequence in $B M O A$. Let $B_{1}$ denote the unit ball of $B M O A$ endowed with the relative weak ${ }^{*}$ topology and let $B_{2}$ be the ball endowed with the topology of uniform convergence on compact sets. Since $H^{1}$ is separable, $B_{1}$ is metrizable. It is clear that $B_{2}$ is metrizable. It then follows from Lemma 1 that the identity map from $B_{1}$ to $B_{2}$ is continuous. Since $B_{1}$ is compact, this map is a homeomorphism.

As an example we mention that $\left(z^{n}\right)$ converges weakly to zero in $V M O A$.

Lemma 2. Let $E=\left\{e^{i t}|| b\left(e^{i t}\right) \mid=1\right\}$. Then

$$
\lim _{n \rightarrow \infty}\left\langle T^{*} b^{n}, z^{n}\right\rangle=|E| \text {. }
$$

Proof. It is enough to note that

$$
\left\langle T^{*} b^{n}, z^{n}\right\rangle=\left\langle b^{n}, b^{n}\right\rangle=\frac{1}{2 \pi} \int_{-\pi}^{\pi}\left|b\left(e^{i \theta}\right)\right|^{2 n} d \theta,
$$

and apply the bounded convergence theorem. 
We now prove the one positive implication between $T$ on $H^{1}$ and $T^{*}$ on VMOA.

Theorem 2. With $E$ as above, if $T^{*}: V M O A \rightarrow V M O A$ is completely continuous, then $|E|=0$ and hence $T$ is completely continuous on $H^{1}$.

Proof. Let $\sigma_{n} b$ denote the $n$th Cesàro mean of $b$, so that $\sigma_{n} b \in C$ and $\left\|\sigma_{n} b\right\|_{\infty} \leq\|b\|_{\infty} \leq 1$. Thus $\left\|\sigma_{n} b\right\|_{*} \leq 1$. If $b_{r}\left(e^{i \theta}\right)=b\left(r e^{i \theta}\right)$, then it is easy to see that $\sigma_{n} b\left(r e^{i \theta}\right)=\sigma_{n} b_{r}\left(e^{i \theta}\right)$, so that for fixed $r,\left|\sigma_{n} b\left(r e^{i \theta}\right)\right| \leq$ $\sup _{\theta}\left|b\left(r e^{i \theta}\right)\right|$. Hence if $\left(n_{k}\right)$ is any increasing sequence of integers, it follows that $\left(\sigma_{n_{k}} b\right)^{k}$ converges uniformly to zero on compact subsets of the unit disk, and hence weakly in VMOA. Since $T^{*}$ is completely continuous, it follows that $\left\|T^{*}\left(\sigma_{n_{k}} b\right)^{k}\right\|_{*} \rightarrow 0$. If $e^{i \theta}$ is a point at which $\left|b\left(e^{i \theta}\right)\right|<1$, then $\left|\sigma_{n_{k}} b\left(e^{i \theta}\right)^{k} \bar{b}\left(e^{i \theta}\right)^{k}\right| \leq\left|b\left(e^{i \theta}\right)\right|^{k} \rightarrow 0$, so

$$
\lim _{k \rightarrow \infty} \frac{1}{2 \pi} \int_{\partial \mathbb{D} \backslash E} \sigma_{n_{k}} b\left(e^{i \theta}\right)^{k} \bar{b}\left(e^{i \theta}\right)^{k} d \theta=0 .
$$

Now assume the $|E|>0$. Then there is a subsequence $\left(\sigma_{n_{k}} b\right)$ which converges to $b$ a.e. By Egoroff's Theorem, there exists $E_{1} \subset E$ with $\left|E_{1}\right|>$ $\left(1-\frac{1}{2}\right)|E|$ such that $\sigma_{n_{k}} b \rightarrow b$ uniformly on $E_{1}$. Hence there exists $k_{1}$ such that $\left|\sigma_{n_{k}} b-b\right|<1$ on $E_{1}$ if $k>k_{1}$. Again, by Egoroff's Theorem, there exists $E_{2}, E_{1} \subset E_{2} \subset E$, with $\left|E_{2}\right|>\left(1-\frac{1}{3}\right)|E|$ such that $\sigma_{n_{k}} b \rightarrow b$ uniformly on $E_{2}$. Hence there exists $k_{2}>k_{1}$ such that $\left|\sigma_{n_{k}} b-b\right|<\frac{1}{2^{2}}$ on $E_{2}$ if $k>k_{2}$. By induction there is a sequence of subsets $E_{1} \subset E_{2} \subset \cdots \subset E$ such that $\left|E_{p}\right|>\left(1-\frac{1}{p+1}\right)|E|$ for each $p$, and such that $\sigma_{n_{k}} b \rightarrow b$ uniformly on $E_{p}$, and a sequence of integers $k_{1}<k_{2}<\cdots$ such that $\left|\sigma_{n_{k}} b-b\right|<\frac{1}{p^{2}}$ on $E_{p}$ if $k>k_{p}$. Hence

$$
\begin{aligned}
\left|1-\left(\sigma_{n_{k_{p}}} b\right)^{p} \bar{b}^{p}\right| & =\left|b^{p}-\left(\sigma_{n_{k_{p}}} b\right)^{p}\right| \\
& =\left|b-\left(\sigma_{n_{k_{p}}} b\right)\right|\left|\sum_{j=0}^{p-1} b^{j}\left(\sigma_{n_{k_{p}}}\right)^{p-1-j}\right| \\
& \leq \frac{1}{p^{2}} \cdot p=\frac{1}{p}
\end{aligned}
$$

on $E_{p}$. Thus

$$
|| E_{p}\left|-\frac{1}{2 \pi} \int_{E_{p}}\left(\sigma_{n_{k_{p}}} b\right)^{p} \bar{b}^{p}\right|<\frac{1}{p}\left|E_{p}\right| \leq \frac{1}{p} .
$$

Since $\left|E \backslash E_{p}\right|<\frac{1}{p+1}$ and $\left|\left(\sigma_{n_{k_{p}}} b\right) \bar{b}\right| \leq 1$, it follows that

$$
|| E\left|-\frac{1}{2 \pi} \int_{E}\left(\sigma_{n_{k_{p}}} b\right)^{p} \bar{b}^{p}\right|<\frac{3}{p} \text {. }
$$

Thus

$$
\lim _{p \rightarrow \infty} \frac{1}{2 \pi} \int_{-\pi}^{\pi}\left(\sigma_{n_{k_{p}}} b\right)^{p} \bar{b}^{p}=|E|
$$

but

$$
\left|\frac{1}{2 \pi} \int_{-\pi}^{\pi}\left(\sigma_{n_{k_{p}}} b\right)^{p} \bar{b}^{p}\right|=\left|\left\langle\left(\sigma_{n_{k_{p}}} b\right)^{p}, T z^{p}\right\rangle\right|=\left|\left\langle T^{*}\left(\sigma_{n_{k_{p}}} b\right)^{p}, z^{p}\right\rangle\right| \leq\left\|T^{*}\left(\sigma_{n_{k_{p}}} b\right)^{p}\right\|_{*},
$$


and $\left\|T^{*}\left(\sigma_{n_{k_{p}}} b\right)^{p}\right\|_{*} \rightarrow 0$, contradicting the assumption that $|E|>0$.

We next construct examples to show that the converse of Theorem 2 is not valid and it is necessary to work out the action of $T^{*}$ on functions in VMOA.

Lemma 3. For $|z|<1$, and $b: \Delta \rightarrow \Delta$, a holomorphic mapping with $b(0)=0$, we have

$$
T^{*} f(z)=\frac{1}{2 \pi} \int_{0}^{2 \pi} \frac{f\left(e^{i \theta}\right)}{1-z \bar{b}\left(e^{i \theta}\right)} d \theta .
$$

Proof. Let $g_{z}(w)=\frac{1}{1-\bar{z} w}$, so that $f(z)=\left\langle f, g_{z}\right\rangle$. Then formally,

$$
T^{*} f(z)=\left\langle T^{*} f, g_{z}\right\rangle=\left\langle f, g_{z} \circ b\right\rangle=\frac{1}{2 \pi} \int_{-\pi}^{\pi} f\left(e^{i \theta}\right) \frac{1}{1-z \bar{b}\left(e^{i \theta}\right)} d \theta,
$$

and this is easily justified for $|z|<1$.

For $1 \leq A<\infty$ let $S_{A}(z)=\frac{z}{A-(A-1) z}$. Then $S_{A}$ is a linear fractional transformation taking 0 to 0 and mapping the unit disk $\mathbb{D}$ onto the disk $\mathbb{D}(a, 1-a)$ of center $a$ and radius $1-a$, where $a=\frac{A-1}{2 A-1}$. A short computation shows that $S_{A} \circ S_{B}=S_{A B}$. In particular, if $T_{S_{A}}^{*}$ is completely continuous and $B>A$, then $T_{S_{B}}^{*}$ is also completely continuous, since $S_{B}=S_{A} \circ S_{B-A}$ and complete continuity is an ideal property. Conversely, if $T_{S_{A}}^{*}$ is not completely continuous, then $T_{S_{B}}^{*}$ is not completely continuous for $1 \leq B \leq A$.

Lemma 4. If $f(z)=z h(z)$, then

$$
T_{S_{A}}^{*} f(z)=\frac{z}{A} h\left(\frac{A-1+z}{A}\right) .
$$

Proof. This follows from Lemma 3 and the Residue Theorem. Indeed,

$$
\begin{aligned}
T_{S_{A}}^{*} f(z) & =\frac{1}{2 \pi} \int_{-\pi}^{\pi} \frac{f\left(e^{i \theta}\right)}{1-z \frac{1}{A e^{i \theta}-(A-1)}} d \theta \\
& =\frac{1}{2 \pi i} \int_{|\zeta|=1} \frac{\zeta-\frac{A-1}{A}}{\zeta-\frac{A-1+z}{A}} \frac{f(\zeta)}{\zeta} d \zeta \\
& =\frac{z}{A} h\left(\frac{A-1+z}{A}\right) .
\end{aligned}
$$

Lemma 5. The operator $T_{S_{2}}^{*}$ is not completely continuous.

Proof. If $f(z)=z h(z)$, it follows from Lemma 4 that

$$
T_{S_{2}}^{*} f(z)=\frac{z}{2} h\left(\frac{1+z}{2}\right) \text {. }
$$

The powers $z^{n}$ form a weakly null sequence of norm one functions in $V M O A$, so it will suffice to estimate the norms of the functions $T_{S_{2}}^{*} z^{n}=\frac{z}{2^{n}}(1+z)^{n-1}$. Let $K_{m}\left(e^{i t}\right)$ denote Fejér's kernel,

$$
K_{m}\left(e^{t}\right)=\sum_{k=-m}^{k=m}\left(1-\frac{|k|}{m+1}\right) e^{i k t} .
$$


Then $K_{m}\left(e^{i t}\right) \geq 0$ and $\frac{1}{2 \pi} \int_{-\pi}^{\pi} K_{m}\left(e^{i t}\right) d t=1$, so $\left\|K_{m}\right\|_{1}=1$. Next, let $V_{m}\left(e^{i t}\right)=2 K_{2 m+1}\left(e^{i t}\right)-K_{m}\left(e^{i t}\right)$. Then $\left\|V_{m}\right\|_{1} \leq 3, V_{m}$ has nonnegative Fourier coefficients, which are equal to 1 for $-m \leq k \leq m$, and has degree $2 m+1$ as a trigonometric polynomial. Finally, let $W_{m}\left(e^{i t}\right)=e^{i(2 m+1) t} V_{m}\left(e^{i t}\right)$. Then $W_{m} \in H^{1},\left\|W_{m}\right\|_{1}=\left\|V_{m}\right\| \leq 3$, and $W_{m}$ has nonnegative Taylor coefficients which are equal to one for $m+1 \leq k \leq 3 m+1$. Suppose $n$ is even and $m=n / 2$. Then $3 m+1 \geq n$, and it follows that

$$
\left\langle T_{S_{2}}^{*} z^{n}, W_{m}\right\rangle \geq \frac{1}{2^{n}} \sum_{k=m}^{n-1}\left(\begin{array}{c}
n-1 \\
k
\end{array}\right)=\frac{1}{2^{n+1}} \sum_{k=0}^{n-1}\left(\begin{array}{c}
n-1 \\
k
\end{array}\right)=\frac{1}{4} \text {. }
$$

Thus

$$
\frac{1}{4} \leq\left\langle T_{S_{2}}^{*} z^{n}, W_{m}\right\rangle \leq\left\|W_{m}\right\|_{1}\left\|T_{S_{2}}^{*} z^{n}\right\|_{*} \leq 3\left\|T_{S_{2}}^{*} z^{n}\right\|_{*} .
$$

Hence $\left\|T_{S_{2}}^{*} z^{n}\right\|_{*} \geq \frac{1}{12}$ for even $n$, and so $T_{S_{2}}^{*}$ is not completely continuous.

\section{FAiluRE OF COMPLETE CONTINUITY}

We have noted that $L^{1}$ has the Dunford-Pettis property and we recall two results of J. Bourgain [B1] to the effect that a bounded linear operator $R$ on $L^{1}$ into a Banach space $Y$ has the Dunford-Pettis property if and only if $R i_{p}$ is a compact operator ( $i_{p}$ is the canonical injection of $L^{p}$ into $L^{1}$ ) for some $1<p \leq \infty$ (or if and only if $R i_{\infty}$ is compact). He also proves that if a bounded linear operator on $L^{1}$ fails the Dunford-Pettis property then there is a measurable subset $\Omega$ of $[0,1]$ and a weakly null sequence $\left(g_{n}\right)$ in $L^{1}$ and $\epsilon>0$ satisfying $|\Omega|>0,\left\|g_{n}\right\|_{1}=1$ with

$$
\limsup _{n \rightarrow \infty}\left\|R\left(f g_{n}\right)\right\| \geq \epsilon\|f\|_{1}
$$

if $f$ is in $L^{1}(\Omega)$ and $f \geq 0$. We have an analogue of this.

Theorem 3. Let $X$ be a separable Banach space and $T: X \rightarrow X$ a bounded linear operator which is not completely continuous but whose adjoint $T^{*}$ is completely continuous. Then there is a sequence $\left(\phi_{n}\right)$ of unit vectors in $X^{*}$ and $\delta>0$ such that $\left(\phi_{n}\right)$ converges to zero weak ${ }^{*},\left(\phi_{n}\right)$ has no weak Cauchy subsequences, and $\left\|T^{*} \phi_{n}\right\| \geq \delta$ for each $n$. Moreover, $T^{*} \phi_{n}$ also converges to zero weak ${ }^{*}$.

Proof. Because $T$ is not completely continuous, there is a weakly null sequence $\left(f_{n}\right)$ in $X$ and an $\epsilon>0$ such that $\left\|T f_{n}\right\|>\epsilon$ for each $n$. For each $n$, the Hahn-Banach Theorem produces $\phi \in X^{*}$ such that $\left\|\phi_{n}\right\|=1$, and $\left|\phi_{n} T f_{n}\right|>\epsilon$. Since $X$ is separable, the unit ball of $X^{*}$ is metrizable in the weak * topology, so $\left(\phi_{n}\right)$ has a weak * convergent subsequence. Thus, passing to a subsequence if necessary, we may assume that $\phi_{n} \stackrel{\mathbf{w}^{*}}{\rightarrow} \phi$. Since $\left(f_{n}\right)$ is weakly null, there is a positive integer $N$ such that $\left|T^{*} \phi f_{n}\right|=\left|\phi T f_{n}\right|<\frac{\epsilon}{2}$ if $n \geq N$. Then

$$
\frac{\epsilon}{2}<\left|\left(\phi_{n}-\phi\right) T f_{n}\right| \leq\|T\| \cdot\left\|\phi_{n}-\phi\right\|
$$

for $n \geq N$. In particular, $\frac{\epsilon}{2\|T\|} \leq\left\|\phi_{n}-\phi\right\| \leq 2$. Let $\psi_{n}=\frac{\phi_{n}-\phi}{\left\|\phi_{n}-\phi\right\|}$ for $n \geq N$. Then $\left\|\psi_{n}\right\|=1, \psi_{n} \stackrel{\mathbf{w}^{*}}{\rightarrow} 0$, and

$$
\left|\psi_{n} T f_{n}\right|>\frac{\epsilon}{2\left\|\phi_{n}-\phi\right\|} \geq \frac{\epsilon}{4}
$$

for $n \geq N$. Let $\delta=\frac{\epsilon}{4}$. Then 


$$
\delta<\left|\psi_{n} T f_{n}\right|=\left|T^{*} \psi_{n} f_{n}\right| \leq\left\|T^{*} \psi_{n}\right\|
$$

for $n \geq N$. In order to complete the proof, assume that $\left(\psi_{n}\right)$ has a weak Cauchy subsequence. Passing to a subsequence if necessary, we may assume that $\left(\psi_{n}\right)$ is itself weak Cauchy. Let $n_{1}=1$. Since $\left(f_{n}\right)$ is weakly null, there exists $n_{2}>n_{1}$ such that $\left|T^{*} \psi_{n_{1}} f_{n_{2}}\right|<\frac{\delta}{2}$. Then

$$
\frac{\delta}{2}<\left|T^{*}\left(\psi_{n_{2}}-\psi_{n_{1}}\right) f_{n_{2}}\right| \leq\left\|T^{*}\left(\psi_{n_{2}}-\psi_{n_{1}}\right)\right\|,
$$

because $\left|T^{*} \psi_{n_{2}} f_{n_{2}}\right|=\left|\psi_{n_{2}} T f_{n_{2}}\right|>\delta$. Choose $n_{3}>n_{2}$ so that $\left|T^{*} \psi_{n_{2}} f_{n_{3}}\right|<\frac{\delta}{2}$. Then again

$$
\frac{\delta}{2}<\left|T^{*}\left(\psi_{n_{3}}-\psi_{n_{2}}\right) f_{n_{3}}\right| \leq\left\|T^{*}\left(\psi_{n_{3}}-\psi_{n_{2}}\right)\right\| .
$$

We can continue inductively to produce $n_{1}<n_{2}<n_{3}<\cdots$ so that $\frac{\delta}{2}<$ $\left\|T^{*}\left(\psi_{n_{k+1}}-\psi_{n_{k}}\right)\right\|$ for all $k$. But $\psi_{n_{k+1}}-\psi_{n_{k}}$ is weakly null, so this contradicts the assumption that $T^{*}$ is completely continuous. Finally, the last observation is obvious.

Remark 2. Since $\left(\psi_{n}\right)$ has no weak Cauchy subsequence, it follows from (the complex version of) Rosenthal's $l_{1}$ Theorem [Ro1], [Dor] that some subsequence of $\left(\psi_{n}\right)$ is equivalent to the usual unit vector basis of $l_{1}$. Passing to this subsequence and reindexing, there is an $\epsilon>0$ such that for any $n$ and scalars $a_{1}, \ldots, a_{n}$ we have

$$
\epsilon \sum_{i=1}^{n}\left|a_{i}\right| \leq\left\|\sum_{i=1}^{n} a_{i} \psi_{i}\right\| \leq \sum_{i=1}^{n}\left|a_{i}\right| .
$$

Of course, the last inequality is free. Hence if $\left|b\left(e^{i t}\right)\right|<1$ a.e. and $T^{*}: V M O A$ $\rightarrow V M O A$ is not completely continuous, there is a sequence $\left(f_{n}\right)$ of functions in $H^{1}$ and positive numbers $\epsilon>0, \delta>0$ such that $\left\|f_{n}\right\|_{1}=1$ and $\left\|f_{n} \circ b\right\|_{1}>$ $\delta$ for all $n$, and such that for any $n$ and scalars $a_{1}, \ldots, a_{n}$ we have

$$
\epsilon \sum_{i=1}^{n}\left|a_{i}\right| \leq\left\|\sum_{i=1}^{n} a_{i} f_{i}\right\|_{1} .
$$

Finally, as we noted, we have been unable to locate general results on the connection between complete continuity of an operator and complete continuity of its adjoint. The following result provides one such connection (and it also yields a proof of one of our earlier theorems).

Proposition 2. Let $X$ be a Banach space with separable dual $X^{*}$ and let $T: X \rightarrow$ $X$ be a completely continuous operator. Then $T^{*}: X^{*} \rightarrow X^{*}$ is also completely continuous.

Proof. Assume that $T: X \rightarrow X$ is completely continuous, but $T^{*}$ is not. Then there is a weakly null sequence $\left(\phi_{n}\right)$ in $X^{*}$ and a $\delta>0$ such that $\left\|T^{*} \phi_{n}\right\|>\delta$ for all $n$. For each $n$, choose $f_{n} \in X$ with $\left\|f_{n}\right\|=1$ such that $\left|T^{*} \phi_{n} f_{n}\right|>$ $\delta$. Let $q: X \rightarrow X^{* *}$ be the canonical imbedding. Since $X^{*}$ is separable, the unit ball of $X^{* *}$ is metrizable in the weak ${ }^{*}$ topology and hence the bounded sequence $\left(q f_{n}\right)$ has a weak * convergent subsequence. Reindexing if necessary we may assume that $\left(q f_{n}\right)$ converges weak ${ }^{*}$ to $F \in X^{* *}$. Let $n_{1}=1$. Since $T^{*} \phi_{n}$ is weakly null, there exists $n_{2}>n_{1}$ such that $\left|T^{*} \phi_{n_{2}} f_{n_{1}}\right|<\frac{\delta}{2}$. Inductively, 
suppose $n_{1}<n_{2}<\cdots<n_{k}$ have been chosen. Choose $n_{k+1}>n_{k}$ so that $\left|T^{*} \phi_{n_{k+1}} f_{n_{k}}\right|<\frac{\delta}{2}$. Let $f_{n_{0}}=0$, and for $k \geq 1$ let $g_{k}=f_{n_{k}}-f_{n_{k}}$. Then $\left(g_{k}\right)$ is weakly null and

$$
\left|T^{*} \phi_{n_{k}} g_{k}\right|=\left|T^{*} \phi_{n_{k}} f_{n_{k}}-T^{*} \phi_{n_{k}} f_{n_{k-1}}\right|>\frac{\delta}{2}
$$

But

$$
\left|T^{*} \phi_{n_{k}} g_{k}\right|=\left|\phi_{n_{k}} T g_{k}\right| \leq\left\|\phi_{n_{k}}\right\|\left\|T g_{k}\right\| \text {. }
$$

and $\left\|T g_{k}\right\| \rightarrow 0$ since $T$ is completely continuous. This is the desired contradiction.

Remark 3. The separability of $X^{*}$ is crucial. Indeed, the identity operator on $l_{1}$ is completely continuous. This is just a restatement of the fact that $l_{1}$ has the Schur property [Di, p. 85]. The adjoint operator is the identity operator on $l_{\infty}$, which is not completely continuous. Similarly, the identity operator on $c_{0}$ is not completely continuous, but its adjoint, the identity operator on $l_{1}$, is.

\section{REFERENCES}

[B] Stefan Banach, Opérations linéaires, Chelsea, New York, 1932.

[B1] J. Bourgain, A characterization of non-Dunford-Pettis operators on $L^{1}$, Israel J. Math. 37 (1980), 48-53.

[B2] — Dunford-Pettis operators on $L^{1}$ and the Radon-Nikodym property, Israel J. Math. 37 (1980), 48-53.

[Co] J. Conway, A course in functional analysis, Springer-Verlag, New York, 1985.

[Di] J. Diestel, A survey of results related to the Dunford-Pettis property, Contemp. Math. 2 (1980), 15-60.

[D] - Sequences and series in Banach spaces, Springer-Verlag, New York, 1984.

[Dor] L. Dor, On sequences spanning a complex $l_{1}$ space, Proc. Amer. Math. Soc. 47 (1975), 515-516.

[DP] N. Dunford and J. T. Schwartz, Linear operators, part I, Wiley-Interscience, New York, 1958.

[Ro1] H. P. Rosenthal, $A$ characterization of Banach spaces containing $l_{1}$, Proc. Nat. Acad. Sci. U.S.A. 71 (1974), 2411-2413.

[Ro2] _ Point-wise compact subsets of the first Baire class, Amer. J. Math. 99 (1977), 803-831.

[Sa1] D. Sarason, Composition operators as integral operators, Analysis and Partial Differential Equations, Marcel Dekker, New York, 1990.

[Sa2] _ Weak compactness of holomorphic composition operators, Lecture Notes in Math., vol. 1511, Springer-Verlag, Berlin, 1990.

[Sh1] J. H. Shapiro, Compact composition operators on spaces of boundary-regular holomorphic functions, Proc. Amer. Math. Soc. 100 (1987), 49-57.

[Sh2] _ The essential norm of a composition operator, Ann. of Math. 125 (1987), 375-404.

[SS] J. H. Shapiro and C. Sundberg, Compact composition operators on $L^{1}$, Proc. Amer. Math. Soc. 108 (1990), 443-449.

[ST] J. H. Shapiro and P. D. Taylor, Compact, nuclear, and Hilbert-Schmidt composition operators on $H^{2}$, Indiana Univ. Math. J. 23 (1973), 471-496.

Department of Mathematics, University of North Carolina, Chapel Hill, North CarOLINA 27599

E-mail address: cima@math.unc.edu

Department of Mathematics, Lamar University, Beaumont, Texas 77710

E-mail address: matheson@math. lamar.edu 